\title{
Intervenciones educativas para la prevención del cáncer cervicouterino
}

\author{
Giselle Riquelme H. ${ }^{1 a}$, Ximena Concha P. 2a, María Teresa Urrutia S. 3a \\ ${ }^{1}$ Matrona, MSc. ${ }^{2}$ Enfermera Matrona, MSc. ${ }^{3}$ Enfermera Matrona, PhD. \\ a Departamento de Salud de la Mujer, Escuela de Enfermería, Facultad de Medicina, Pontificia Universidad Católica de \\ Chile.
}

\section{RESUMEN}

Antecedentes: El cáncer cervicouterino (CC) se ha convertido en los últimos años en el cáncer más común en la mujer. La detección precoz a través del screening de PAP ha sido insuficiente, siendo indispensable buscar nuevas estrategias para prevenirlo, una de las cuales es la incorporación de intervenciones educativas. Objetivos: Conocer las intervenciones educativas destinadas a la prevención del CC e identificar sus características principales. Método: Se realizó búsqueda bibliográfica en MEDLINE, ProQuest, Scielo y metabuscador Tripdatabase, seleccionándose para su análisis, 15 artículos que respondían al objetivo planteado. Análisis: Las intervenciones educativas utilizadas para la prevención del CC abordan temas como el conocimiento general sobre CC, PAP y HPV, las creencias sobre CC, y las actitudes preventivas entre otros. Los folletos educativos y las sesiones de discusión son las metodologías más utilizadas en este tipo de intervenciones, midiéndose la efectividad de éstas a través de la aplicación de pre y post test, ya sea inmediatamente después de la intervención, o a lo largo del tiempo. Se observa que este tipo de intervenciones educativas son efectivas para mejorar aspectos como el nivel de conocimiento en la población y la adquisición de conductas preventivas (adherencia a la toma de PAP). Conclusión: Considerando los beneficios de las intervenciones educativas en la prevención del CC, se hace fundamental ampliar su utilización, no olvidando que su empleo implica la prevención de la enfermedad, pudiéndose evitar muertes de mujeres jóvenes y el aumento de la carga de salud por enfermedad de un país.

\section{PALABRAS CLAVES: Educación, cáncer cervicouterino, prevención}

\section{SUMMARY}

Background: Cervical cancer has become in recent years the most common cancer in women. Early detection through screening of PAP has been insufficient, being indispensable to search for new strategies to prevent it, one of which is the incorporation of educational interventions. Objective: To educational interventions aimed at preventing cervical cancer and identify its main features. Method: Literature search was conducted in MEDLINE, ProQuest, Scielo and Tripdatabase, selecting for analysis, 15 research articles that reflected the purpose stated. Analysis: Educational interventions used to address cervical cancer prevention topics such as general knowledge about cervical cancer, PAP and HPV, cervical cancer beliefs, attitudes, preventive against this disease, among others. The educational brochures (pamphlets) and discussion sessions are the methodologies used in these interventions, measuring the effectiveness of these through the application of pre and post test, either immediately after surgery, or over time. Note that this type of educational interventions are effective in improving aspects such as the level of knowledge in the population and the 
acquisition of preventive behavior (adherence to PAP). Conclusion: Considering the benefits of educational interventions in the prevention of cervical cancer, it is essential to expand its use, not forgetting that their job involves the prevention of the disease, being able to prevent deaths of young women and increasing health burden of disease of a country.

\section{KEY WORDS: Education, cervical cancer, prevention}

\section{INTRODUCCIÓN}

A nivel mundial, el cáncer cervicouterino (CC) se ha convertido en los últimos años en el cáncer más común en la mujer, diagnosticándose cerca de 500.000 nuevos casos cada año $(1,2)$. En nuestro país es la quinta causa de muerte por cáncer en la mujer con una tasa de mortalidad de 8,5 por 100.000 mujeres (3).

La detección precoz a través del screening de Papanicolaou (PAP) es una estrategia efectiva para prevenir el CC y reducir la mortalidad por este cáncer (4). No obstante, las mujeres muestran baja adherencia a la toma de este examen, lo que se ve reflejado en un nivel de cobertura que alcanza el $66 \%$ (2001), bajo el nivel óptimo determinado por la Organización Mundial de la Salud (OMS) de $80 \%$. (2-5). Considerando este elemento se hace indispensable buscar nuevas estrategias para prevenir este cáncer, tales como la incorporación de intervenciones educativas que complementen la utilizada actualmente.

El objetivo de este estudio es conocer las intervenciones educativas destinadas a la prevención del CC, descritas en la literatura, e identificar las características principales de ellas.

\section{METODOLOGÍA}

Se realizó una búsqueda en las bases de datos Medline/Pubmed, ProQuest, Scielo y el metabuscador Tripdatabase durante los meses de marzo y abril del 2011. Las palabras claves utilizadas fueron: intervention and cervical cancer, prevention and cervical cáncer, cervical screening, education and cervical cancer, y sus respectivas traducciones cuando se realizó la búsqueda en bases de datos en español.

Se encontró un total de 26 artículos, seleccionando aquellos que cumplían con una antigüedad no mayor a 10 años que incluían la temática de interés. Finalmente, se utilizaron 15 investigaciones para esta revisión.

A continuación se presenta el análisis del tema para esta revisión que abarcó los siguientes pun- tos: contenidos de una intervención educativa en $\mathrm{CC}$, metodologías utilizadas en la intervención, medición de la efectividad de la intervención y resultados obtenidos.

\section{ANÁLISIS DEL TEMA}

Contenidos de una intervención educativa en cáncer cervicouterino. Las intervenciones educativas que se utilizan en la prevención del CC contemplan una amplia gama de contenidos relacionados al tema, desde las características generales del cáncer, a la forma de contagio (relacionados al HPV) y detección precoz del mismo.

En relación al CC los contenidos a considerar deben incorporar aspectos epidemiológicos de la enfermedad, factores de riesgo, cuadro clínico, forma de detección y tratamiento (6-15), haciendo énfasis en que esta enfermedad es prevenible $(6,10,11)$.

La información sobre el examen de PAP suele incorporarse a la sección de detección precoz dentro de las generalidades del CC. Sin embargo, es importante mencionar que aspectos tales como el propósito del examen $(10,11)$ o el detalle específico de cómo se realiza el procedimiento (10), en algunos casos fueron contenidos destacados dentro de la intervención.

Por su parte, los contenidos acerca del HPV constituyen un capítulo aparte a la hora de abordar el CC, que va desde la historia virológica del HPV (16) a datos específicos sobre la vacuna (8). En términos generales existe coincidencia en que los contenidos básicos sobre HPV deben considerar la historia natural y formas de contagio del virus $(8,11,16-19)$, la relación entre el HVP y el CC $(8,11,16,17)$, formas de prevención (conductas preventivas) $(16,19)$ y la vacuna para $\operatorname{HPV}(8,17,18)$.

Metodologías utilizadas en la intervención. El tipo de metodología a utilizar es quizás el elemento central de una intervención y su efectividad determina el éxito final de la misma. Para esta revisión entenderemos como metodología todo aquel elemento, herramienta o tecnología empleada en la realización de una intervención educativa. 
Son variadas las metodologías que se han empleado en las intervenciones educativas para la prevención del CC siendo las más comunes los folletos educativos y las sesiones de discusión.

Los folletos educativos en sus distintas modalidades (dípticos y trípticos) son un material de bajo costo, con información acotada al tema central y escrito en lenguaje sencillo, que se utilizan como material único o complementario a otras metodologías (reforzando contenidos), y que pueden ser entregados antes, durante $o$ al finalizar la intervención $(6,7,9,11-13,16)$.

Las sesiones de discusión (también conocidas como preguntas/respuestas) son reuniones interactivas con grupos pequeños de mujeres en las cuales se abordan los distintos temas en torno al $\mathrm{CC}$. En estas sesiones se suelen conjugar distintas metodologías, desde el planteamiento de preguntas por parte de un moderador que motiva a la discusión $(6,7,9,10,15)$. La incorporación de folletos (entregados al inicio o final de la sesión de discusión) $(7,9)$; la presentación de contenidos teóricos (utilizando Microsoft office power point o rotafolio) $(7,10)$; la representación de una situación real (roleplay) $(6,10)$; o la conjunción de todas las anteriores, manteniendo como eje central la discusión en torno al tema.

Otras metodologías que se utilizan en las intervenciones educativas para la prevención del CC son el role-play (que suele ser complementaria a otra metodología) $(6,10)$, los grupos focales $(8,17)$, campañas en medios de comunicación (programas de radio, mensajes en televisión o diarios) $(7,14,15)$ y visitas domiciliarias (20).

Medición de la efectividad de la intervención educativa. La aplicación de un test de preguntas es la estrategia más ampliamente utilizada para medir la efectividad de una intervención educativa en prevención de CC $(6,7,10-12,15,16,19)$. Son test en su mayoría de preguntas cerradas (ej. verdadero y falso), auto administrado, con un número variable de preguntas que depende de la cantidad de contenidos que componen cada intervención $(6,7,12,16,19)$.

Se realiza en primera instancia una medición inicial que consiste en la aplicación del test previo a la realización de la intervención (pre-test) con el fin de conocer el estado basal de la temática a abordar en la intervención, ya sea nivel de conocimientos, creencias respecto al tema, actitudes o conductas de prevención $(6,7,14,19)$. Una vez aplicado el test basal se procede a medir las variables previamente determinadas por el investigador (post-test) en mediciones secuenciales que pueden ser inmediatamente después de realizada la intervención
$(10,20)$, dentro del primer mes (ej. dos semanas) (16), dos meses $(7)$, tres meses $(11,12,15,16,19)$, cuatro meses $(15,20)$, seis meses $(6) \mathrm{u}$ ocho meses (17). En algunos casos se puede realizar más de una medición (ej. inmediata a la intervención y a los cuatro meses) (20).

En algunos estudios es utilizaron entrevistas estructuradas y semiestructuradas $(11,13,14)$ realizadas posterior a la intervención y en las cuales no se realizó medición basal, requiriéndose un tiempo promedio para el total de entrevistas de tres meses $(11,13,14)$.

Resultados obtenidos. Las siguientes variables fueron los elementos principales medidos en las distintas investigaciones revisadas: aumento de la toma de PAP en las mujeres del estudio $(6,7,20)$; nivel de conciencia (6) y conocimiento sobre CC y $\operatorname{PAP}(6,7,10,12,14,15)$; creencias y actitudes frente al CC $(10,15)$; nivel de conocimiento sobre HPV (16-18); percepción de riesgo para HPV $(11,18) ; y$ cambios en el nivel de conocimiento, actitudes y factores sicosociales que impiden las prácticas preventivas en salud $(6,15)$. En algunas intervenciones se midieron variables específicas de la educación impartida (ej. nivel de entendimiento de la intervención realizada) (16).

En aquellos casos en que se utilizaron metodologías cualitativas de investigación $(9,13)$, las variables correspondieron a las categorías que emergieron del análisis de los datos y que coinciden con las variables medidas en las investigaciones cuantitativas.

Respecto a la variable aumento de la toma de PAP en las mujeres del estudio, las investigaciones muestran un aumento significativo en la toma del examen de PAP (6) o bien en la intención de realizarse el screening (7) después de la intervención educativa. Sólo en uno de los estudios no se observó diferencias (20).

El nivel de conocimiento general sobre CC y PAP se ve mejorado en aquellos grupos sometidos a una intervención educativa en torno al tema $(6,7,10,12,14-16)$, observándose mejoras puntuales en elementos tales como lugares donde realizarse el PAP o características de este examen (8-14-15), síntomas asociados al CACU (12), y factores de riesgo para $\mathrm{CC}(6,12,15)$.

En el nivel de conocimiento sobre HPV, uno de los estudios arrojó como resultados que la población intervenida poseía un nivel básico de conocimiento sobre este tema, el cual no variaba posterior a la intervención en forma significativa (16). En otro de los estudios, de carácter cualitativo, se observó que las mujeres, ya sea adolescentes o adultas, tienen un limitado conocimiento sobre el HPV, observándose 
un importante nivel de desconocimiento en relación a la forma de transmisión del virus, aspectos que mejoran posterior a la intervención educativa, así como también la aceptación parental de la vacuna (17), elemento que también es referido en otro estudio (18).

En la percepción de riesgo para HPV los estudios mostraron que posterior a la intervención educativa aumentó la percepción de riesgo en el grupo de mujeres jóvenes (16 a 24 años), no observándose cambios entre aquellas mujeres que se tomaban el PAP versus las que no lo realizaban (11). Por su parte aumentó la intención de colocarse la vacuna para el HPV (18). En uno de los estudios que abordaron esta temática no se observaron diferencias significativas en el grupo que recibió una intervención educativa (12).

Finalmente, en la variable cambios en el nivel de conocimiento, actitudes y factores psicosociales que impiden las prácticas preventivas en salud, los resultados muestran un cambio positivo en la percepción de autoeficacia en la población que recibió la intervención educativa (6) y cambio de conducta entre la negación a la toma de PAP a la aceptación del mismo (15).

Cabe destacar que las mejoras en las distintas variables obtenidas, posterior a la realización de la intervención, se observaron tanto en la medición inmediatamente después de la intervención como en las mediciones realizadas en meses posteriores a ésta.

\section{DISCUSIÓN}

Las intervenciones educativas para la prevención del CC son una metodología que se ha comenzado a incorporar como estrategia de prevención en los últimos años, debido a su bajo requerimiento de recursos (humanos y materiales), como la necesidad de aumentar el nivel educacional en la población objetivo, a sabiendas que una población educada en salud mejora sus conductas preventivas.

Por su parte, los diferentes cambios que se han ido observado en torno a CC, tales como la incorporación del virus HPV como su agente precursor y las formas de prevención de su infección como la incorporación de vacunas, así como la constante negativa por parte de las mujeres a realizarse el examen del PAP, desafían a los profesionales de la salud a buscar estrategias factibles de ser aplicadas en la población que garanticen el éxito considerando recursos limitados.

No obstante, es fundamental reconocer las características que debe tener una intervención educativa para la prevención del CC. Por ejemplo, la conjunción de los contenidos a ser incorporados en una intervención educativa dependerá de la población a la que está dirigida la intervención, observándose que los contenidos acerca de la vacuna son más utilizados a la hora de educar adolescentes versus mujeres adultas (16-19), mientras que aspectos como la toma del PAP, específicamente la forma en que se realiza el procedimiento, están dirigidos a la población objetivo de la toma de este examen (mujer adulta) $(7,6,14,15,20)$. Subsecuentemente, se debe considerar las falencias principales de la población, es decir, la elección de los contenidos dependerá del diagnóstico de la situación que se realice previo a la realización de la intervención.

Con los actuales avances tecnológicos se podría pensar que las estrategias apuntan a la utilización de dichas tecnologías a la hora de educar a la población sobre un tema en particular. Sin embargo, es importante considerar los recursos con los cuales se cuenta, las características de la población a intervenir (ej, nivel educacional) y la factibilidad de la aplicación de la tecnología sobre el tema que se desea educar. Las sesiones de discusión $(6,7,9,10,15)$ y los folletos educativos parecen ser las estrategias más ampliamente utilizadas $(6-9,11$ $13,16)$, debido probablemente a que su bajo costo las hace accesibles y asegura la entrega de la información necesaria. No obstante, es de considerar que su utilización suele no ser la única metodología a emplear, considerando la combinación de éstas entre sí o con otras metodologías, según los recursos disponibles. La importancia final radica en la elección de una metodología que garantice la retención de los contenidos entregados en la población beneficiada.

Un elemento fundamental a considerar en la realización de una intervención educativa es la forma en que será medida la efectividad de ésta. El uso de pre y post test es una estrategia ampliamente utilizada, la que puede ser aplicada tanto inmediatamente después de la intervención como a través del tiempo. En esta última modalidad la medición a los 3 meses de realizada la intervención es la más común, y se observó sólo un estudio con un tiempo mayor (ocho meses) (17).

Respecto a los resultados, las variables medidas corresponden a los contenidos abordados en las intervenciones educativas, tales como conocimientos sobre CC y HPV, conocimientos sobre PAP, entre otros. En todos los estudios se observó una mejora significativa entre la medición basal y la medición post intervención, lo que conlleva a reafirmar la efectividad de una intervención educativa como estrategia exitosa a la hora de intervenir 
en la población. Destaca el hecho que los estudios analizados no evaluaron la satisfacción de los participantes con respecto a la intervención realizada.

\section{CONCLUSIÓN}

Considerando los beneficios de las intervenciones educativas en la prevención del CC, es fundamental ampliar su utilización ya sea en otros escenarios de intervención, así como el pilotaje de nuevas metodologías en aprovechamiento de la tecnología avanzada en educación, aplicada en salud. No debemos olvidar que su empleo implica la prevención de la enfermedad, pudiéndose evitar muertes de mujeres jóvenes y el aumento de la carga de salud por enfermedad de un país.

\section{REFERENCIAS}

1. Leung Sh, Leung I. Cervical cancer screening: knowledge, health perception and attendance rate among Hong Kong Chinese women. Int $\mathrm{J}$ Womens Health 2010:2 221-8.

2. Sepúlveda P, González F, Napolitano C, Roncone E, Cavada G. Cáncer de cuello uterino: sobrevida a 3 y 5 años en Hospital San José. Rev Chil Obstet Ginecol 2008;73:151-4.

3. Donoso E, Cuello M. Mortalidad por cáncer en la mujer chilena. Análisis comparativo entre los años 1997 y 2003. Rev Chil Obstet Ginecol 2006;71:10-6.

4. Urrutia MT, Araya A, Poupin L. ¿Por qué las mujeres no se toman el Papanicolaou? Respuestas entregadas por los profesionales del programa cáncer cervicouterino-AUGE del Servicio de Salud Metropolitano Sur Oriente. Rev Chil Obstet Ginecol 2010;75(5): 282-9.

5. Donoso E, Cuello M, Villarroel L. Reducción de la mortalidad por cáncer cervicouterino en Chile, 1990-2003. Rev Chil Obstet Ginecol 2006;71:307-12.

6. Mishra S, Luce P, Baquet C. Increasing PAP smear utilization among Samoan women: results from a community based participatory randomized trial. J Health Care Poor Underserved 2009;20:85-101.

7. Lam T, McPhee S, Mock J, Wong Ch, Doan H, Nguyen $\mathrm{T}$, et al. Encouraging Vietnamese-American Women to obtain PAP test trough lay health worker outreach and media education. J Gen Intern Med 2003;18:516-24.

8. Wong Ping L. HPV information needs, educational messages and channel of delivery preferences: views from developing country with multiethnic populations. Vaccine 2009;27:1414-15.

9. Vivilaki V, Romanidou A, Theodorakis PN, Lionis C. Are health education meetings effective in recruiting women in cervical screening programmes? An innovative and inexpensive intervention from the island of Crete. Rural Remote Health 2005:376. Hallado en: http://www.rrh. org.au/publishedarticles/article_print_376.pdf

10. Tejeda S, Thompson B, Coronado G, Rees J. A cervical cancer curriculum for Hispanic adolescent in rural High Schools: a pilot study. J Health Care Poor Underserved 2006;17:734-44.

11. Marlow L, Waller J, Wardle J. The impact of HPV information on perceived risk of cervical cancer. Cancer Epidemiol Biomarkers Prev 2009;18:373-6.

12. Wrigth K, Kuyini YA, Faduyille FA. Community education on cervical cancer amongst market women in an urban area of Lagos, Nigeria. Asian Pac J Cancer Prev 2010;11:137-40.

13. Hunter J. Cervical cancer educational pamphlets: do they miss the mark for Mexican inmigrant women's needs? Cancer Control 2005:12 suppl 2:42-50.

14. Perkins R, Langrish S, Stern LJ, Simon C. A community-based education program about cervical cancer improves knowledge and screening behavior in Honduran women. Pan Am J Public Health 2007; 22:187-93.

15. Mock J, McPhee S, Nguyen T, Wong Ch, Doan H, Lay $\mathrm{K}$, et al. Effective lay health worker outreach and media based education for promoting cervical cancer screening among Vietnamese American women. Am J Public Health 2007;97:1693-700.

16. Wetzel C, Tissot A, Kollar L, Hillard P, Stone R, Kahn J. Development of an HPV educational protocol for adolescents. J Pediatr Adolesc Gynecol 2007;20:281-7.

17. Cooper S, Bernard D, McCaffery K, Brotherton J, Garland S, Skinner R. "Is cancer contagious?": Australian adolescent girls and their parents: making the most of limited information about HPV and HPV vaccination. Vaccine 2010;28:3398-08.

18. Cox D, Cox A, Sturn Lynne, Zimet G. Behavioral Interventions to increase HPV vaccination acceptability among mothers of young girls. Health Psychology 2010;29:29-39.

19. Lambert E. College students' knowledge of human papilomavirus and effectiveness of a brief educational intervention. J Am Board Fam Pract 2001;14:178-83.

20. Chalapati W, ChumworathayiB. Can a Home-Visit Increase Pap smear screening in Samlien, Khon Kaen, Thailand? Asian Pac J Cancer Prev 2007;8:119-23. 